\title{
WEAK LAWS FOR DEPENDENT SUMS
}

\author{
WILLIAM L. STEIGER
}

\begin{abstract}
A general weak law of large numbers for sums $S_{n}=X_{1}+\cdots+X_{n}$ is proved. That is, without assuming the existence of any moments, and allowing any sort of dependence structure, conditions are given for $S_{n} / n \rightarrow 0$ in probability; the conditions are not necessary. However they are sufficient for a much stronger statement, namely that $S_{v_{n}} / \nu_{n} \rightarrow 0$ in probability in many cases where positive, integer-valued random variables $v_{n} \rightarrow \infty$.
\end{abstract}

Introduction. Let $\left\{X_{i}\right\}$ be a sequence of random variables on a probability space $(\Omega, \mathscr{A}, p)$ with partial sums $S_{n}=X_{1}+\cdots+X_{n}$. For reals $0<a_{n} \rightarrow \infty, X_{n}={ }^{p} o\left(a_{n}\right)$ means that $X_{n} / a_{n} \rightarrow 0$ in probability. The weak law of large numbers holds when $S_{n}={ }^{p} o(n)$.

When the $X_{n}$ are independent the weak law is well understood. Indeed, Kolmogoroff [1] gave a set of conditions, (C), that are necessary and sufficient for $S_{n}={ }^{p} O(n)$. However without independence, the results are less striking. The best one seems to be that $f\left(S_{n}\right) \equiv E\left(S_{n}^{2} /\left(n^{2}+S_{n}^{2}\right)\right) \rightarrow 0$, $n \rightarrow \infty$, is necessary and sufficient for the weak law, a fact that states the obvious; $d(X, Y) \equiv f(X-Y)$ defines a metric for the (equivalence classes of almost surely equal) random variables on $\Omega$ whose topology is that of convergence in probability. Because it is difficult to know when $d\left(S_{n}, 0\right) \rightarrow 0, n \rightarrow \infty$, this paper explicates the weak law for dependent sums.

It turns out that a conditional version of (C) is sufficient but not necessary for the weak law; this is Proposition 1. A corollary is that when integer random variables $v_{n}>0$ are sufficiently well-behaved, then also $S_{v_{n}}={ }^{p} o\left(v_{n}\right)$, a weak law for random sums; the $v_{n}$ need not be independent of the $X_{n}$.

This work began with the proof of the corollary and depended on the truth of Proposition 1. However the obvious sources ([2] and [4]) made no mention of the weak law in the present context, a surprising fact that necessitated proving this simple result.

The results. For each $n \geqq 1$ let $\mathscr{F}_{n} \subseteq \mathscr{F}_{n+1} \subseteq \mathscr{A}$ be a sigma field of subsets of $\Omega$ containing the Borel field $B\left(S_{n}\right)$ generated by $S_{n}$ and put

Received by the editors March 19, 1973.

AMS (MOS) subject classifications (1970). Primary 60F05, 60F99, 60G45.

Key words and phrases. Weak law of large numbers, dependent random variables, random sums.

(c) American Mathematical Society 1973 
$\mathscr{F}_{0} \equiv\{\varnothing, \Omega\}$. For $A \in \mathscr{A}, I_{A}$ denotes the characteristic function of $A$ and $\bar{A}=\Omega \mid A$. Finally let $E_{j, n}=\left\{\omega \in \Omega:\left|X_{j}\right| \leqq n\right\}$. We prove the following result.

Proposition 1. $S_{n}={ }^{p} o(n)$ if

$$
\begin{gathered}
\sum_{j=1}^{n} p\left(\bar{E}_{j, n}\right) \rightarrow 0, \\
\sum_{j=1}^{n} E\left(X_{j} I_{E_{j, n}} \mid \mathscr{F}_{j-1}\right) \equiv \sum_{j=1}^{n} c_{j}={ }^{p} o(n), \\
\sum_{j=1}^{n} E\left[\left(X_{j} I_{E_{j, n}}-c_{j}\right)^{2}\right]=o\left(n^{2}\right) .
\end{gathered}
$$

Proof. Put $X_{j}^{\prime}=X_{j} I_{E_{j, n}}, Y_{j}=X_{j}^{\prime}-E\left(X_{j}^{\prime} \mid \mathscr{F}_{j-1}\right), S_{j}^{\prime}=X_{1}^{\prime}+\cdots+X_{j}^{\prime}$ and $T_{j}=Y_{1}+\cdots+Y_{j}$. Since $B\left(S_{j}^{\prime}\right) \subseteq \mathscr{F}_{j},\left\{T_{i}, \mathscr{F}_{i}\right\}$ is a martingale.

Given an integer $n>0$ and numbers $\varepsilon, \delta>0$, let $A_{n}=\left\{\left(\omega \in \Omega:\left|S_{n}\right|>\delta n\right\}\right.$, and $A_{n}^{\prime}=\left\{(1) \in \Omega:\left|S_{n}^{\prime}\right|>\delta n\right\}$. Clearly

$$
\begin{aligned}
p\left(A_{n}\right) & =p\left(A_{n} \cap\left\{S_{n}=S_{n}^{\prime}\right\}\right)+p\left(A_{n} \cap\left\{S_{n} \neq S_{n\}}^{\prime}\right\}\right. \\
& =p\left(A_{n}^{\prime}\right)+p\left(A_{n} \cap\left\{S_{n} \neq S_{n}^{\prime}\right\}\right) \leqq p\left(A_{n}^{\prime}\right)+p\left(S_{n} \neq S_{n}^{\prime}\right) \\
& \leqq p\left(A_{n}^{\prime}\right)+\sum_{j=1}^{n} p\left(\bar{E}_{j, n}\right)
\end{aligned}
$$

and, by (1), the second term can be made less than $\varepsilon / 3$ for $n$ large enough. Now

$$
\begin{aligned}
p\left(A_{n}^{\prime}\right) & =p\left(\left|T_{n}+S_{n}^{\prime}-T_{n}\right|>\delta n\right) \\
& \leqq p\left(\left|T_{n}\right|>\delta n / 2\right)+p\left(\left|S_{n}^{\prime}-T_{n}\right|>\delta n / 2\right)
\end{aligned}
$$

and since $S_{n}^{\prime}-T_{n}=\sum_{j=1}^{n} c_{j}={ }^{p} O(n)$ by (2),

$$
p\left(A_{n}^{\prime}\right)<p\left(\left|T_{n}\right|>\delta n / 2\right)+\varepsilon / 3
$$

for large enough $n$.

By Tchebycheff $p\left(\left|T_{n}\right|>\delta n / 2\right) \leqq 4 E\left(T_{n}^{2}\right) /(n \delta)^{2}$ and since $\left\{T_{j}, \mathscr{F}_{j}\right\}$ is a martingale, $E\left(T_{n}^{2}\right)=\sum_{j=1}^{n} E\left(Y_{j}^{2}\right)$. Hence, because of (3) and the preceding remark,

$$
p\left(\left|T_{n}\right|>\delta n / 2\right)<\varepsilon / 3
$$

for large enough $n$. Combining (4), (5), (6), $p\left(A_{n}\right)<\varepsilon$ for all sufficiently large $n$, which, because $\varepsilon, \delta$ are arbitrary, proves the asserted proposition.

REMARK. Let $\left\{S_{i}\right\}$ be a sequence of independent, symmetrically distributed random variables and define $X_{1}=S_{1}, X_{n+1}=S_{n+1}-S_{n}, n \geqq 1$. The following examples show that none of the conditions of the proposition are necessary. 
First, let $p\left(S_{n}=n / \log (n+1)\right\}=\frac{1}{2}-\frac{1}{2} n^{-1}$ and $p\left\{S_{n}=e^{n+1}\right\}=\frac{1}{2} n^{-1}, n \geqq 1$, so that $S_{n}={ }^{p} o(n)$. However $\sum_{i=1}^{n} p\left\{\bar{E}_{i, n}\right\} \geqq \sum(4 i+1) /\left(i^{2}+1\right)>1$ for large $n$, the latter sum extending over $[\log n]+1 \leqq i \leqq n$. Thus (1) fails; $\left\{S_{n}\right\}$ need not be tail equivalent to sums of truncates for the weak law to hold.

Next, let $p\left\{S_{n} / n=1 / \log (n+1)\right\}=\frac{1}{2}$ so that $S_{n}=o(n)$ almost surely. However when $j>7,\left|X_{j}\right|<j$ so that $X_{j} I_{E_{j, n}}=X_{j}$ when $1 \leqq j \leqq n$ and $n>7$. Therefore

$$
\sum_{i=1}^{n} E\left(X_{i} I_{E_{i, n},} \mid \mathscr{F}_{i-1}\right)=\sum_{i=1}^{n} E\left(X_{i} \mid \mathscr{F}_{i-1}\right)=-\sum_{i=1}^{n-1} S_{i} .
$$

Since

$$
W_{n} \equiv n^{-2} \sum_{i=1}^{n} E\left(S_{i}^{2}\right)=n^{-2} \sum_{i=1}^{n}\left(\frac{i}{\log (i+1)}\right)^{2} \geqq K>0 \text { for large } n,
$$

$-\sum_{i=1}^{n-1} S_{i}={ }^{p} o(n)$ is false, $W_{n} \rightarrow 0$ being necessary. Thus (2) fails; centering truncates at conditional means need not, on the average, have a negligible effect for the weak law of large numbers to hold.

Finally, the same example shows that (3) is also not necessary for the weak law. Under the above notation, $n^{2} W_{n}$ is equal to the expression on the left-hand side of (3). By the foregoing this is not $o\left(n^{2}\right)$, since $W_{n} \geqq K>0$.

Let $v_{n}>0$ be integer-valued random variables on $(\Omega, \mathscr{A}, p)$ not necessarily mutually independent nor independent of the $X_{n}$. In view of the preceding proposition it is natural to expect that $S_{v_{n}}={ }^{p} o\left(v_{n}\right)$, as long as $v_{n} \rightarrow \infty$ in some appropriate way. The following result, motivated by work in [3], shows that this is indeed the case.

Corollary. Let $0<a_{n} \rightarrow \infty$ be reals and $\nu_{n}>0$ be integer-valued random variables on $(\Omega, \mathscr{A}, p)$ that satisfy

$$
v_{n} / a_{n} \stackrel{\mathscr{L}}{\longrightarrow} F, \quad F(0)=0,
$$

where $\stackrel{\mathscr{L}}{\rightarrow}$ denotes convergence in law and $F$ is a distribution function. Then $S_{v_{n}}={ }^{p} o\left(v_{n}\right)$ under the conditions of the proposition.

Proof. $S_{n}={ }^{p} o(n)$ by the proposition. Let $\varepsilon, \delta>0$ be given and choose $0<x<y<\infty, x, y$ continuity points of $F$, so that $F(y)-F(x)>1-\varepsilon / 6$. Defining

$$
B_{n}=\left\{\omega \in \Omega: v_{n} / a_{n} \in(x, y]\right\},
$$

and noting that both $\left|F(x)-p\left\{v_{n} \mid a_{n} \leqq x\right\}\right|<\varepsilon / 12$ and $\left|F(y)-p\left\{v_{n} \mid a_{n} \leqq y\right\}\right|<$ $\varepsilon / 12$ are true for large $n$ because of (7), $p\left(B_{n}\right)=p\left\{v_{n} / a_{n} \leqq y\right\}-F(y)+$ $F(y)-F(x)+F(x)-p\left\{v_{n} / a_{n} \leqq x\right\}>F(y)-F(x)-\varepsilon / 6>1-\varepsilon / 3$ for large $n$. 
Consequently, defining $D_{n}=\left\{\omega \in \Omega:\left|S_{v_{n}}\right| \geqq \delta v_{n}\right\}$,

$$
p\left(D_{n}\right)<\varepsilon / 3+p\left(D_{n} \cap B_{n}\right)
$$

for large $n$.

Let $j_{n}=\left[x \dot{a}_{n}\right], k_{n}=\left[y a_{n}\right]+1([\cdot]$ is the greatest integer function on $R \rightarrow N)$, and note that $v_{n} \in\left[j_{n}, k_{n}\right]$ on $B_{n}$. Hence, the inclusion

$$
D_{n} \cap B_{n} \subseteq B_{n} \cap\left(\left\{\left|S_{j_{n}}\right| \geqq \delta v_{n} / 2\right\} \cup\left\{\left|S_{v_{n}}-S_{j_{n}}\right| \geqq \delta v_{n} / 2\right\}\right)
$$

holds which, along with (8), means that

$$
p\left(D_{n}\right)<\varepsilon / 3+p\left(B_{n} \cap\left\{\left|S_{j_{n}}\right| \geqq \delta v_{n} / 2\right\}\right)+p\left(B_{n} \cap\left\{\left|S_{v_{n}}-S_{j_{n}}\right| \geqq \delta v_{n} / 2\right\}\right) \text {. }
$$

When $n$ is large, the middle term is $\left\langle\varepsilon / 3\right.$ because $S_{n}={ }^{p} o(n)$ and $\nu_{n} \geqq j_{n}$ on $B_{n}$; i.e., $p\left(D_{n}\right)<2 \varepsilon / 3+p\left(B_{n} \cap\left\{\left|S_{v_{n}}-S_{j_{n}}\right|>\delta v_{n} / 2\right\}\right)$.

Using the notation of the proof of the proposition, fix $n$ and put

$$
\begin{array}{ll}
X_{j}^{\prime}=X_{j} I_{E_{j, k_{n}}}, & Y_{j}=X_{j}^{\prime}-E\left(X_{j}^{\prime} \mid \mathscr{F}_{j-1}\right), \\
S_{j}^{\prime}=X_{1}^{\prime}+\cdots+X_{j}^{\prime}, & T_{j}=Y_{1}+\cdots+Y_{j}, \quad j=1, \cdots, k_{n} .
\end{array}
$$

On $B_{n},\left\{\left|S_{v_{n}}-S_{j_{n}}\right| \geqq \delta v_{n} / 2\right\} \subseteq\left\{\bigcup_{*}\left(\bar{E}_{j, k_{n}}\right)\right\} \cup\left\{\left|S_{v_{n}}^{\prime}-S_{j_{n}}^{\prime}\right| \geqq \delta v_{n} / 2\right\}, \cup_{*}$ being the union over $j \in\left[j_{n}, k_{n}\right]$, and $\left\{\left|S_{v_{n}}^{\prime}-S_{j_{n}}^{\prime}\right| \geqq \delta v_{n} / 2\right\} \subseteq\left\{\left|S_{v_{n}}^{\prime}-T_{v_{n}}+T_{j_{n}}-S_{j_{n}}^{\prime}\right| \geqq\right.$ $\left.\delta v_{n} / 4\right\} \cup\left\{\left|T_{v_{n}}-T_{j_{n}}\right| \geqq \delta v_{n} / 4\right\}$. Combining these inclusions with the last statement of the preceding paragraph, and using (1) and (2), there results, for large enough $n$,

$$
p\left(D_{n}\right)<8 \varepsilon / 9+p\left(B_{n} \cap\left\{\left|T_{v_{n}}-T_{j_{n}}\right| \geqq \delta v_{n} / 4\right\}\right) .
$$

Since $B_{n} \cap\left\{\left|T_{v_{n}}-T_{j_{n}}\right| \geqq \delta v_{n} \mid 4\right\} \subseteq\left\{\sup \left(\left|T_{i}-T_{j_{n}}\right| \geqq \delta j_{n} / 4, j_{n} \leqq i \leqq k_{n}\right)\right\}$, Kolmogoroff's inequality gives

$$
p\left(D_{n}\right)<8 \varepsilon / 9+16 E\left(T_{k_{n}}-T_{j_{n}}\right)^{2} /\left(\delta^{2} j_{n}^{2}\right) .
$$

Finally, since $j_{n}^{-1} \leqq 4 y x^{-1} k_{n}^{-1}$ and because $\left\{\boldsymbol{T}_{j}, \mathscr{F}_{j}\right\}$ is a martingale, (3) applies to show $p\left(D_{n}\right)<\varepsilon$ for large $n$, as required for $S_{v_{n}}={ }^{p} o\left(v_{n}\right)$.

\section{REFERENCES}

1. A. N. Kolmogoroff, Über die Summen durch den Zufall bestimmter unabhängiger Grössen, Math. Ann. 99 (1928), 309-319: Math. Ann. 102 (1929), 484-488.

2. P. Lévy, Théorie de l'addition des variables aléatoires, Gauthier-Villars, Paris, 1954.

3. J. Mogyorodi, A remark on limiting distribution for sums of a random number of independent random variables, Rev. Roumaine Math. Pures Appl. 16 (1971), 551-557. MR 44 \#6011.

4. P. Révész, The laws of large numbers, Probability and Math. Statist., vol. 4, Academic Press, New York, 1968. MR 39 \#6391.

Centre de Recherches Mathématiques, Université de Montréal, Box 6128, Montréal 101, Québec, Canada 